\title{
A Surgical Planning and Guidance System for High Tibial Osteotomies
}

\author{
C. Y. Tso* \\ R. E. Ellis* \\ J. Rudan ${ }^{\dagger}$ \\ M. M. Harrison ${ }^{\dagger}$ \\ * Department of Computing and Information Science \\ $\dagger$ Department of Surgery
}

Queen's University at Kingston, Ontario, Canada

\begin{abstract}
A three-dimensional pre-surgical planner and image-guided surgery system for high tibial osteotomy has been developed. The planning software permits a surgeon to establish the tibial axes, plan the corrective osteotomy, and visualize both the preoperative state and the predicted postoperative state in three dimensions. The parameters that describe the placement and orientation of the osteotomy resection planes can be transmitted to an accompanying guidance system that allows the surgeon to accurately perform the planned procedure. Tests were performed in vitro to compare the accuracy of the computer-enhanced technique to the traditional technique, and an in vivo pilot study has been initiated. The computer-enhanced technique produced a significant reduction in the error of correction and is simple to use while performing the surgical procedure. The work indicates that three-dimensional planning and surgical performance of high tibial osteotomy is essential to the accuracy of the procedure.
\end{abstract}

\section{Rationale and Objectives}

An osteotomy is a surgical procedure to realign a bone in order to change the biomechanics of a joint, especially to change the force transmission through a joint. Theses procedures must be performed accurately, not only to create the desired geometries but also to provide biological environments for rapid and effective healing. In particular, the high tibial osteotomy (HTO) is a corrective surgical procedure in which the upper part of the tibia is resected so that the lower limb can be realigned. The most common form of HTO is the closing wedge osteotomy, in which the realignment is achieved by removing a lateral wedge of bone from the proximal tibia.

High tibial osteotomies are indicated for the relatively young and active patient who has painful unicompartmental knee arthrosis, especially an arthrosis due to an early stage of osteoarthritis. The most common surgical alternative to the procedure is a total knee arthroplasty (TKA) or unicompartmental arthroplasty. TKA has a typical survival rate of $90 \%$ at 15 years after the procedure, but the polyethylene articulating surfaces generate wear particles that lead to loosening or failure and subsequent revision. By contrast, HTO preserves the joint's original cartilaginous surfaces and corrects the fundamental mechanical problem of the knee. This advantage of HTO is especially important to the young active patient, for whom an arthroplasty has a greater probability of ear- 
lier failure than is likely for an older inactive patient. Economically, the cost of an osteotomy is approximately $1 / 3$ that of an arthroplasty.

The major difficulty with HTO is that the outcome is not predictable, and the major suspected reason for the unpredictability is that it is difficult to attain the desired correction angle. Numerous long-term clinical studies have shown that osteotomy results are improved if the femoral-tibial alignment angle is between $7^{\circ}$ and $13^{\circ}$ of varus alignment $[19,13]$. Immediate postoperative results show a large variability - by averaging the variances of reported results, calculation shows that the overall standard deviation of angular correction is $2.5^{\circ}$ for HTO performed with jig systems and $5.6^{\circ}$ for those without the use of jigs[ $[7,16$, $15,17,10,14]$. Significant changes in the angle of correction after operation can be observed, which can be attributed to fragment mobility. Recent results have suggested that proper patient selection, more careful planning, and more precise execution of the osteotomy can produce a longer-lasting HTO $[12,18]$. For example, an extensive clinical review[20] reports on 128 knees that were studied for up to 15 years, finding a revision rate was only $10.9 \%$.

\subsection{The Traditional Technique for High Tibial Osteotomy}

The most common form of HTO is the closing wedge osteotomy, popularized by Coventry in 1973 [3]. The wedge is first planned on a frontal-plane standing Xray by drawing a wedge of the desired correction angle, where the wedge's upper plane is parallel to the tibial plateau and the lower plane is above the tibial tubercle. Ideally, the wedge will produce a hinge of cortical bone approximately $2-5 \mathrm{~mm}$ in thickness.

Upon surgical exposure of the proximal tibia, the correction is mapped to the bones of the patient via a ruler or a jig system. The surgery is then performed either freehand or with assistance of Kirschner wires (K-wires) as cutting guides. Intraoperative fluoroscopic X-ray is often employed for verification before and during the procedure. Technical difficulties often arise from the use of fluoroscopy, such as image-intensifier nonlinearities and distortions that compromise accuracy, and parallax errors that can provide misleading angular and positional guidance.

\subsection{A Computer Technique for High Tibial Osteotomy}

The major contribution of computer assisted surgery to HTO has so far been in preoperative planning, whereby the surgeon is provided with a prediction of postoperative alignment or loading. One such system, developed by the Tsumura group[23], used a two-dimensional static biomechanical model originally proposed by Coventry and Insall[4, 11]. The other preoperative planner, developed by Chao et al., used a two-dimensional rigid-body spring model to simulate the forces across the articular surfaces $[1,2,9]$. It also attempted to account for ligamentous effects, and simulated two-dimensional dynamic loading.

A drawback of these approaches is that planning and simulation of the three-dimensional osteotomy are carried out in only two dimensions. There are many inherent problems in executing a two-dimensional plan in three-dimension space. For example, tilting of the saw blade in the anterior/posterior direction 
is not controlled by the traditional technique. Further, if the axis for closing the osteotomy is not perpendicular to the frontal plane, an unintended inter$\mathrm{nal} /$ external rotation of the foot is induced.

We submit that the premature failures and large variability in HTO are due to: (1) the inability of current planning methods to address the fact that HTO is a three-dimensional procedure; and (2) the incapability of manual surgeries to produce the planned amount of axis shift accurately. It is insufficient to plan a three-dimensional surgery using the traditional two-dimensional X-ray information. Also, substantial spatial reasoning and surgical experience are required to accurately cut a three-dimensional wedge through the limited surgical exposure.

Our approach to solving these problems is to use computer assistance to achieve accurate three-dimensional corrections. A surgical enhancement system has been developed that facilitates preoperative planning in three-dimensional space. This three-dimensional plan can then be executed in an image-guided surgery system. The inherent precision in the image-guided surgery system is expected to lead to better reliability, consistency and speed in HTO than can be achieved by the traditional techniques.

The goal was to reduce the incidence of the common postoperative problems. With a three-dimensional guidance and planning system it should be possible to eliminate, or greatly reduce the probability of (a) incorrect valgus alignment, (b) incorrect proximal/distal location of the osteotomy, and (c) inadvertent internal/external foot rotation. If proximal-fragment fracture is due to proximity of the osteotomy to the joint, especially because of inadvertent anterior/posterior sloping of the proximal resection, then the probability of fracture should be reduced. Finally, if the post-osteotomy fixation is also planned and guided, the danger of fixation into the joint articulation should also be reduced.

\section{Computer-Assisted Planning and Guidance}

The computer-assisted high tibial osteotomy was performed in nine steps:

1. Three or more radio-opaque markers were inserted into the tibia under local anesthesia, placed slightly distal to the tibial tubercle.

2. $\mathrm{Cr}$ images of the knee were acquired from the femoral condyles to below the tibial tubercle, using axial mode slices of $3 \mathrm{~mm}$ width spaced $2 \mathrm{~mm}$ apart.

3. A three-dimensional surface model of the tibia was reconstructed from the CT images by isosurface extraction.

4. With the isosurface model, and data from standardized weight-bearing radiographs, the surgeon used preoperative planning software to plan the procedure. Given the desired correction in the sagittal and frontal planes, the planner simulated a closing-wedge osteotomy. The surgeon could visually inspect the three-dimensional position of the resection planes and adjust the planes as desired.

5. Intraoperatively, the pre-implanted fiducial markers were located in three dimensions with a precision mechanical arm. 
6. The coordinate system common to the CT scan, the computerized tibia model, and the planned osteotomy were mapped onto the coordinate system of the patient by means of a fiducial registration algorithm [5].

7. The accuracy-critical step of the surgical procedure was performed by attaching a drill to the locating device. A full-screen computer animation displayed the relative positions of surgical instruments superimposed on the planned resection planes, with several screen updates per second. Referring to the online computer graphics, the surgeon then inserted two K-wires into the proximal tibia for each resection plane.

8. The wires were then used as saw guides to perform the osteotomy in a manner similar to the traditional Coventry technique [3]. Osteoclasis was used to complete the formation of a medial hinge.

9. After the bone wedge was removed and the tibia adjusted to the new angulation, standard step staples were used to immobilize the fragments and the surgical field was closed.

\subsection{A Preoperative Planning System}

The closing-wedge osteotomy is rotation about a cortical bone hinge, which when modeled as rotation about an axis in space makes the procedure particularly suited to computer-assisted planning. The planning process is linear and well structured, so the planner was implemented as a wizard-based system in which each window had text describing the step and a graphical interface for effecting that step. The preoperative planning system was implemented in $\mathrm{X} /$ Motif, and its major function was to allow the surgeon to specify two resection planes on a graphical model of a tibia.

The triangulated isosurface model of the tibia was first loaded and displayed. The user selected the tibial anatomical axis and the transverse axis, from which the system computed the frontal axis. The second step was selection of the tibial plateau, which was rendered as a plane that could obscure or be obscured by the proximal tibial surface. The user had control over the varus/valgus tilt, the anterior/posterior tilt, and the proximal/distal placement of where the plateau plane intersected the anatomical axis. Typically the surgeons viewed the superimposed axes and plateau carefully to ensure that they fully understood the anatomy of the individual patient.

The third and final step was selection of the resection planes. The user moved the proximal plane down to an appropriate level (usually $10-14 \mathrm{~mm}$ ) and then specified the correction angle of the wedge. The angle could be measured from a pre-operative plain X-ray film or could be found as an adjustment to the tibialplateau/tibial-shaft angle automatically calculated by the system. The user could translate the hinge axis in proximal/distal and medial/lateral directions, and could rotate it about the internal/external axis.

The most powerful computation performed in the planning system was a simulation of the result of the osteotomy in the third step. The two resection planes intersected the model to produce four components: the proximal fragment, the distal fragment, the lateral wedge, and the medial hinge. The system discarded 
the wedge, rotated the distal fragment to contact the proximal fragment, and "stretched" the hinge component with an affine transformation to provide a smooth rendering of the medial cortex. This stretching was completed by retriangulation of the faces that had been split by intersection with the resection planes.

The original tibia with superimposed resection planes was displayed on one side of the monitor, and on the other side was the predicted result including pre-operative and predicted post-operative axes. The images could be visualized from any viewpoint, to facilitate understanding of the full three-dimensional consequences of the osteotomy. Because the tibial cortex was modeled as a triangular mesh, which allowed a desktop UNIX workstation running OpenGL to render each virtual surgery within one or two seconds. Figure 1 shows a sample completed plan for a plastic tibial model used in a laboratory study.

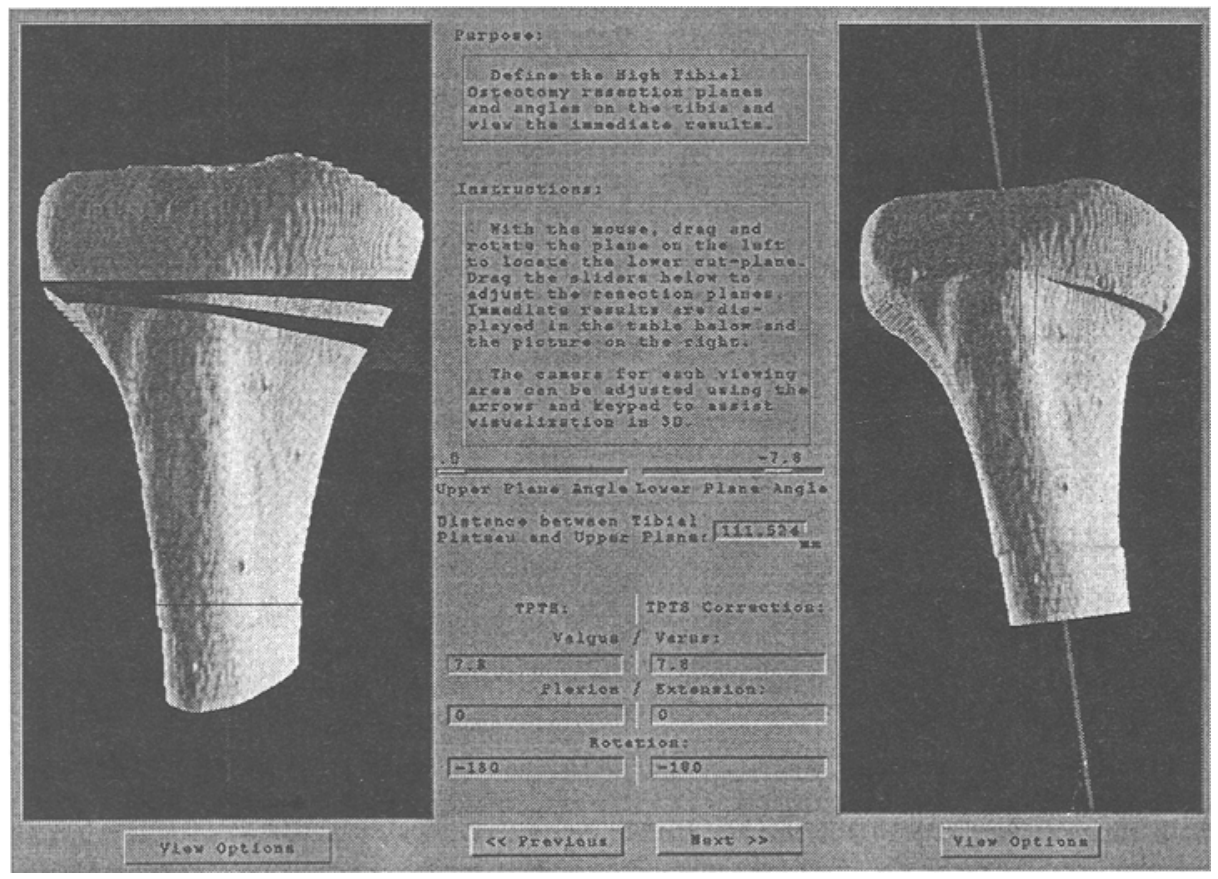

Fig. 1. A preoperative planning system for high tibial osteotomy. The system displays the preoperative and postoperative models of the tibia at real time, as well as the three anatomical angles as changed by the simulated surgery.

The software program guided the user through the steps of preoperative planning. Control of objects in all three dimensions was primarily by means of a mouse, with transformations smoothly rendered by interpolating rotations as quaternions $[8,21,22]$. The osteotomy's geometry was rendered visually and the rotational parameters were displayed as the Grood-Suntay parameters of valgus/varus, internal/external rotation, and flexion/extension [6]. The user could 
adjust the osteotomy's correction via a mouse, or enter values via a keyboard, to achieve the desired correction.

\subsection{An Image-Based Guidance System}

The image guidance system was composed of software running on a desktop UNIX workstation (SUN Microsystems, Sunnyvale CA) and a mechanical localizer (FARO Technologies, Lake Mary, FL). Registration was accomplished by touching three or more pre-implanted fiducial markers with the localizer, then calculating the least-squares rigid transformation between localizer coordinates and CT coordinates [5]. With registration accomplished, it was possible to transform any model or action represented in one coordinate system to the other coordinate system. The surgeons found that the most helpful information was (a) rendering transverse, frontal, and sagittal planes from CT volumetric data, with the location and orientation of the surgical instrument superimposed in color on the gray-scale images, and (b) using solid modeling to render the three-dimensional surface model of the tibia, models of the resection planes, and a three-dimensional model of a virtual surgical instrument. Additional guidance information was available digitally, as required by the surgeon, by means of simple queries to the software. Figure 2 shows the screen layout of the guidance system.

\section{Laboratory Tests}

The laboratory tests were mock surgeries performed on plastic bone models (Pacific Research Laboratories, WA). Prior to testing, the bone models were prepared for registration and scanned with CT. Four registration markers were inserted into accurately milled holes on the antero-lateral surface of each model. The positions were selected such that the markers would be within a realistic surgical exposure and would also be located only on the distal fragment of the tibia after osteotomy.

To facilitate resection each bone model was fixed in a special jig that held the tibia model at a $45^{\circ}$ angle from the horizontal. Black closed-cell foam masked the proximal part of the bone model, to simulate the visual obstructions created by soft tissue that appear clinically. After the wedge was removed, the distal and proximal fragments of the tibia were held together by strong adhesive (Shoe Goo) and with woodworking staples delivered from a staple gun.

Thirty mock surgeries were performed, with intended corrections of $9^{\circ}, 14^{\circ}$, and $19^{\circ}$. The tasks were to (a) perform the osteotomy with fluoroscopic guidance of the saw blade followed by conventional cutting and osteoclasis, and (b) perform the osteotomy by computer-enhanced planning and guidance of pin placement followed by conventional cutting and osteoclasis. For each task each angular correction was performed five times in a randomized sequence, alternating tasks (a) and (b).

Subsequently, the wedge angles were measured. These measurements took advantage of the presence in the models of a transverse hole approximately $15 \mathrm{~mm}$ inferior to the tibial plateau; this hole is parallel to the Cartesian $\mathbf{X}$ axis 


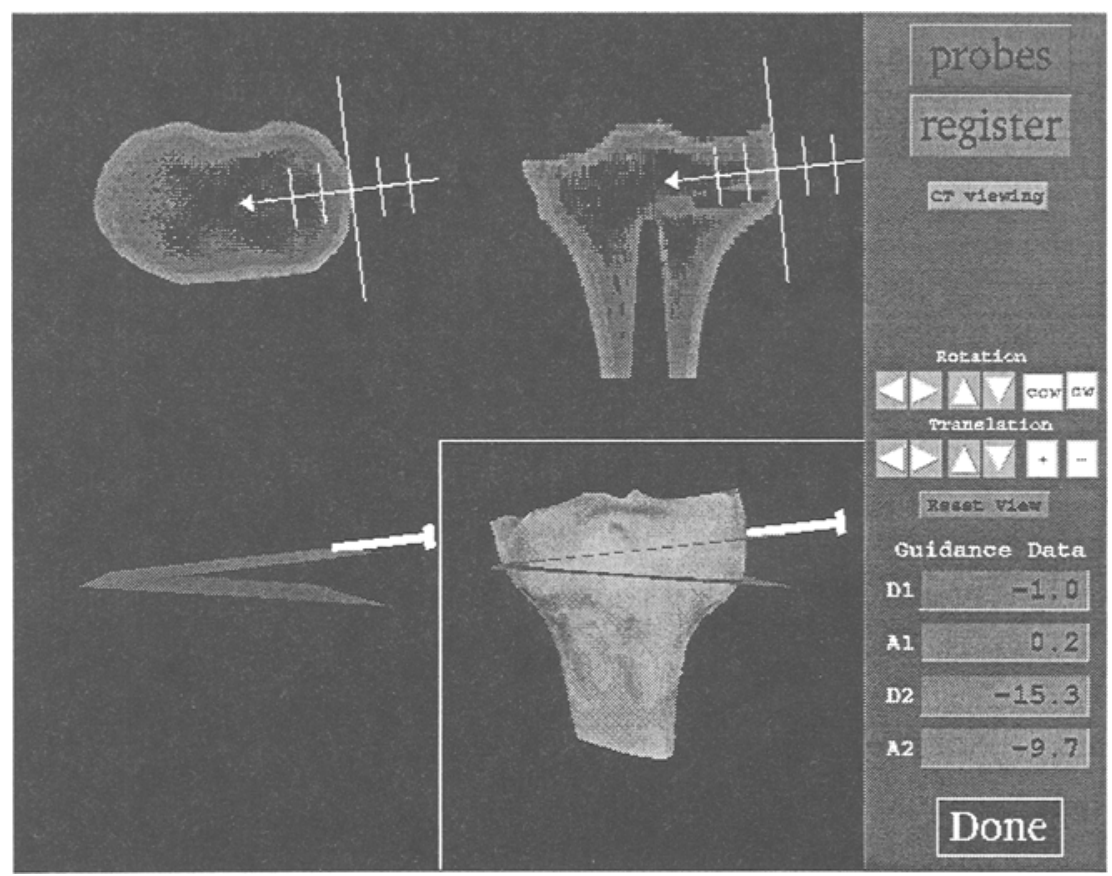

Fig. 2. An image-based guidance system for orthopedic surgery. The surgery software displays the position of the surgical instrument in several reconstructed CT planes, and also shows the tibial model, the planned resection planes, and a virtual surgical instrument.

of the Grood-Suntay coordinate system [6]. The wedges were placed in a jig that fixed the wedges via the transverse hole, and surface points were collected from the superior wedge surface using a precision mechanical arm (Faro Technologies, FL). Varus/valgus angulations were calculated as the total rotation about the Cartesian $\mathbf{Y}$ axis of the Grood-Suntay coordinate system of the tibia.

\subsection{Laboratory Results}

The surgeons found that the two major pieces of software - the 3-D preoperative planner and image-based guidance system - provided an appropriately user-friendly interface. The modeling of preoperative and postoperative alignment gave them an unprecedented control and preview of the surgical procedure. The information provided by the image-based guidance system was very useful, in that they easily installed the guide wires by looking solely at the screen (as they do when performing an arthroscopic procedure).

The test osteotomies were analysed by examining the wedge angles. For traditional and computer-enhanced techniques, the mean and standard deviations of the differences between the intended varus/valgus angle and the achieved varus/valgus angle were computed. Results are shown in Table 1. According to the classic F-test, the reduction in standard deviations are significant to a $95 \%$ 
confidence level. Differences in the mean errors are not statistically significant, and in both sets are very low.

Table 1. Summary of Laboratory Test Results Errors are the difference between the measured varus/valgus angle and the intended angle. The range is the difference between the largest and smallest error.

\begin{tabular}{|c|c|c|c|c|}
\hline Wedg & Errors & raditiona & Wedge Errors & iter-H \\
\hline Mear & d. Dev. & Range & Mean Std. Dev. & Range \\
\hline$-0.4^{\circ}$ & $2.3^{\circ}$ & $8.4^{\circ}$ & $0.1^{\circ}$ & $3.7^{\circ}$ \\
\hline
\end{tabular}

There were no training effects observed in the trials, i.e. the errors were evenly distributed through time. The errors were larger for larger correction angles, which is not a surprising result.

A particularly interesting observation was the accurate formation of the osteotomy hinge when operating with computer assistance. In every trial the hinge was between $1 \mathrm{~mm}$ and $3 \mathrm{~mm}$ in thickness. When operating in the traditional way, with fluoroscopic guidance of the saw blade, the hinge could be non-existent (totally mobile fragments) or up to $5 \mathrm{~mm}$ in thickness.

\section{Clinical Trials}

Clinical tests involved five patients, all having arthrosis confined to the medial compartment. Each patient was preoperatively instrumented with titanium-alloy markers $1.9 \mathrm{~mm}$ diameter, $3.5 \mathrm{~mm}$ length; the markers were the screws of softtissue anchors (Wright Medical) and were inserted percutaneously into the anterolateral aspect of the proximal tibia under local anesthesia. Each patient was then imaged with Roentgen stereogrammetric analysis (to validate the absolute accuracy of the intraoperative registration), with Questor Precision Radiographs (to establish clinical axes in weight-bearing stance), and with axial-mode computed tomography (to provide accurate three-dimensional images of the anatomy and the markers). Instrumentation and imaging were approximately one week prior to surgery. No infection arose from the marker implantation and the patients reported only minor discomfort.

Image preparation and preoperative planning were conducted in a manner identical to the methods used in the laboratory trials. During surgery the patient's lower affected leg was held in a specially constructed holder that could rigidly hold the leg in angles from full extension to over $90^{\circ}$ flexion. Marker identification was simple, and little preparation of the bone surface or surrounding soft tissues was required to expose the tiny markers. Intraoperative registration and drilling of the two guide wires required a maximum of 6 minutes, compared to a minimum of 12 minutes for typical fluoroscopically guided wire insertion. Resection, reduction, and fixation with step-staples were conducted in the traditional manner. Postoperative plain X-ray films were taken to assess the osteotomy and to ensure that the staples did not intrude into joint.

For each patient, the intraoperative evaluation of the procedure was that the medial hinge was correctly placed and of the correct thickness. The resected 
bone surfaces were visually smooth and flat. Technically each osteotomy was not only acceptable but was judged to be between very good to excellent. The postoperative radiographic examination in each case showed very good to excellent bone-surface contact and no fixation problems. Figure 3 shows a representative fluoroscopic image of a reduced and stapled osteotomy, which has excellent mating of the cut surfaces.

Traditional radiographic analysis was conducted for the four patients whose pre-operative and post-operative $\mathrm{X}$-ray films were available. The pre-operative and post-operative tibial-plateau/tibial-shaft angles were calculated, and their differences were compared to the intended corrections that had been established using the pre-operative planning system. Table 2 summarizes these results.

\section{Discussion}

The major contribution of this work has been the development of software that supports preoperative planning of a closing-wedge high tibial osteotomy in three dimensions, and accompanying software that guides the surgeon to achieve the desired result. Work by other investigators, even with the use of computers, planned in only two dimensions. It is possible that the inability of two-dimensional planning to control for all of the angulation variables has contributed to the reputation of high tibial osteotomy as unpredictable.

Table 2. Pre-operative, post-operative, and intended tibial-plateau/tibial-shaft correction angles, and the resulting error, measured from frontal X-ray films. Complete films were unavailable for the first patient.

\begin{tabular}{|c|ccrr|r|}
\hline \multicolumn{2}{|c|}{ Tibial-plateau/tibial-shaft angles, in degrees } \\
\hline Patient & Pre-op & Post-op & Correction Goal & Error \\
2 & 4.0 varus 6.5 valgus & 10.5 & 9.0 & +1.5 \\
3 & 5.5 varus 7.0 valgus & 12.5 & 11.0 & -1.5 \\
4 & 4.0 varus 5.5 valgus & 9.5 & 11.0 & -1.5 \\
5 & 1.0 varus 5.5 valgus & 6.5 & 8.0 & -1.5 \\
\hline
\end{tabular}

The fundamental problem with planning in only the frontal plane is the implicit assumption that the hinge axis is always perpendicular to the frontal plane. The intended kinematics of this plan will be reproduced in the patient only if this edge of closure is exactly as dictated. When the hinge axis deviates from being perpendicular to the frontal plane, extension/flexion and internal/external rotations are inevitably introduced. Elementary $3 \mathrm{D}$ geometry can be used to show that that a small deviation in the location of the hinge axis can easily lead to $10^{\circ}$ or more unintended internal-external rotation of the foot, which in turn can produce abduction or adduction moments on the knee during normal daily activities. Such unintended effects may contribute to a poor biomechanical environment that does not adequately treat the arthrosis from which the patient suffers.

An important clinical advantage of the computer-assisted technique is the accurate placement of the medial hinge. With simple measurements it is relatively easy to control the thickness of the lateral portion of the wedge, but it is much more difficult to place the apex of the wedge with a millimeter or two 


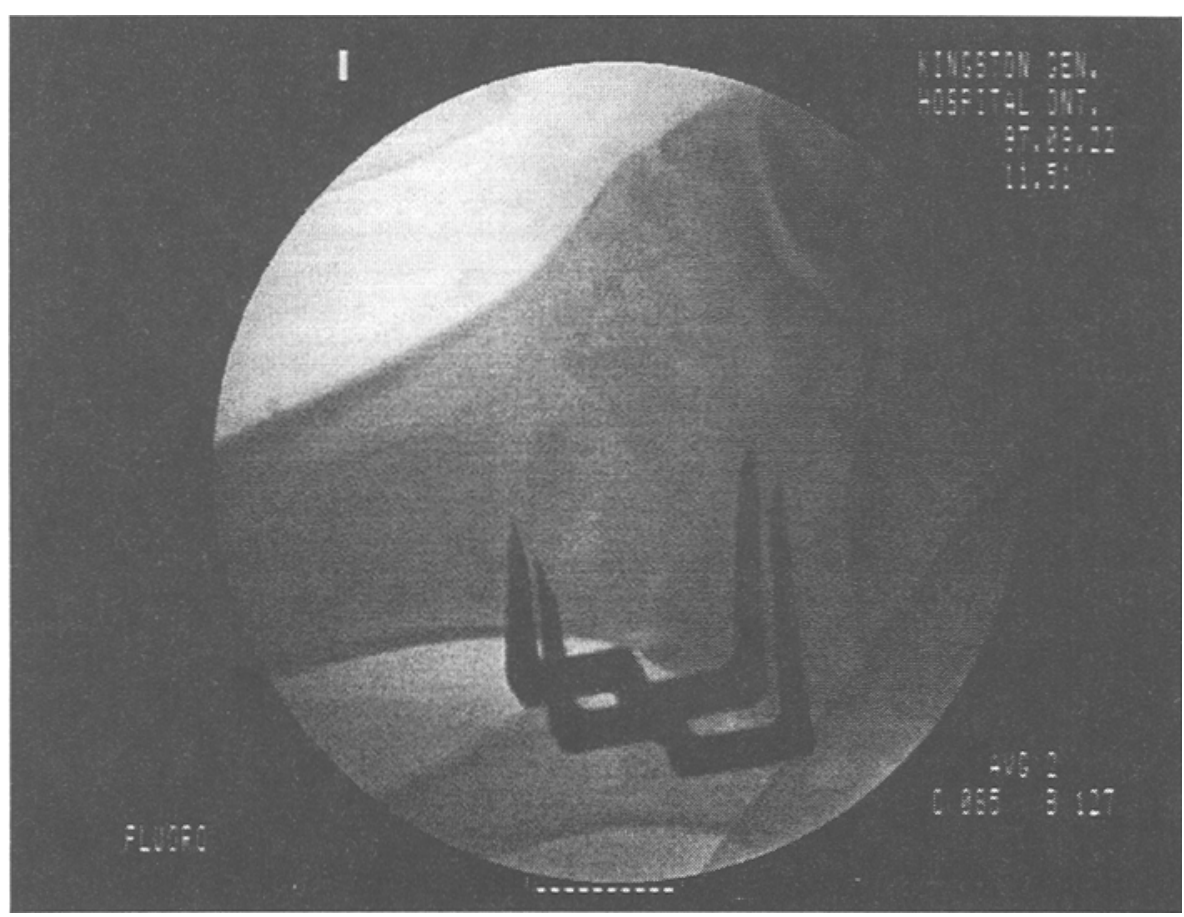

Fig. 3. Fluoroscopic inage of a completed computer-enhanced osteotomy.

of the medial tibial cortex. Jig systems can control the wedge angle, but again the placement of the apex is difficult to ensure even with fluoroscopic imaging. Apex placement is important because small lateral misplacement of the position of the wedge apex can produce a relatively large overcorrection - the small medial changes are propagated across the width of the tibia. If the wedge apex is placed too far medially then medial soft tissues can be disrupted, decreasing the stability of the osteotomy during healing.

The laboratory results show that the angle of the removed lateral wedge can be controlled to an accuracy at least as good as the best jig system. Because the location of the medial cortical hinge can also be controlled, it is expected that computer-enhanced osteotomy will provide superior outcomes than the current course of treatment offers. Use of the computer-assisted HTO system has clarified the importance of incorporating all three dimensions in the planning and execution of this type of osteotomy. In particular, the laboratory tests have revealed some pitfalls that arose from neglecting three-dimensional aspects of osteotomy techniques, and shown that the standard deviation of angular error can be half that of the traditional technique.

The clinical results are in a pilot-study stage but are promising. To date the maximum radiologically measured error is smaller than the standard deviation of the best jig systems. We will continue to recruit patients into our study, and also will follow the progress of the cohort to determine the quality of bone union and retainment of alignment that result from the computer-enhanced technique. 
A shortcoming of the traditional and our computer-enhanced techniques is that neither accurately controls the flexion/extension correction or the internal/external rotation. Flexion/extension can be controlled by use of additional wires, or by determining an additional control point on each resection plane for the initial phase of the saw cut. The computer-assisted technique can also be modified to provide the surgeon with real-time feedback of the foot rotation, so that a desired overall limb alignment can be achieved. This is not trivial to do with a single mechanical arm for location sensing, but is relatively straightforward if optoelectronic position sensors are employed. It may also be possible to track the saw and/or an osteotome so that the surgeon can gain greater control of the osteotomy in the posterior aspect of the tibia, which is not readily seen from an anterolateral surgical approach.

This initial attempt at three-dimensional osteotomy planning concentrated on the development of a three-dimensional environment with geometric simulations. Future work could include biomechanical simulation accounting for the ligaments and for predicted medial/lateral displacement of the joint by normal walking forces. The high tibial osteotomy is a particular use of the closing-wedge osteotomy and the current system could be adapted for application in other anatomical regions. The closing-wedge osteotomy is only one of many corrective orthopedic procedures that could benefit from computer-assisted planning and image-guided surgical techniques.

\section{Acknowledgments}

This research was supported in part by the Natural Sciences and Engineering Research Council of Canada and by Communications and Information Technology Ontario (an Ontario Centre of Excellence). We are grateful to Gordon Goodchild for his careful programming of the guidance system and his assistance in performing the studies. Patricia McAllister and Cameron McLeod coordinated the clinical studies and assisted in marker implantation.

\section{References}

1. E. Y. S. Chao and F. H. Sim. "Computer-aided preoperative planning in knee osteotomy". Iowa Orthopaedic Journal, 15:4-18, 1995.

2. E. Y. S. Chao, J. D. Lynch, and M. J. Vanderploeg. "Simulation and animation of musculoskeletal joint system". Journal of Biomechanical Engineering, 115(4B):562568, November 1993.

3. M. B. Coventry. "Osteotomy about the knee for degenerative and rheumatoid arthritis". Journal of Bone and Joint Surgery [Am], 55(1):23-48, January 1973.

4. M. B. Coventry. "Upper tibial osteotomy for osteoarthritis". Journal of Bone and Joint Surgery [Am], 67(7):1136-1140, 1985.

5. R. E. Ellis, S. Toksvig-Larsen, and M. Marcacci. "Use of a biocompatible fiducial marker in evaluating the accuracy of computed tomography image registration". Investigative Radiology, 31(10):658-667, October 1996.

6. E. S. Grood and W. J. Suntay. "A joint coordinate system for the clinical description of three-dimensional motions: application to the knee". ASME Journal of Biomechanical Engineering, 105:136-144, May 1983. 
7. B. Hagstedt, O. Norman, T. H. Olsson, and B. Tjornstrand. "Technical accuracy in high tibial osteotomy for gonarthrosis". Acta Orthopaedica Scandinavica, 51(6):963970, December 1980.

8. J. C. Hart, G. K. Francis, and L. H. Kauffman. "Visualizing quaternion rotation". ACM Transactions on Graphics, 13(3):256-276, 1994.

9. A. D. Hassen and E. Y. S. Chao. High Tibial Osteotomy, volume 2 of Knee Surgery, pages 1121-1134. Williams and Wilkins, Baltimore, 1994.

10. A. A. Hofmann, R. W. B. Wyatt, and S. W. Beck. "High tibial osteotomy. Use of an osteotomy jig, rigid fixation, and early motion versus conventional surgical technique and cast immobilization". Clinical Orthopaedics and Related Research, 271:212-217, October 1991.

11. J. N. Insall, M. D. Joseph, and C. Msika. "High tibial osteotomy for varus gonarthrosis: A long term follow-up study". Journal of Bone and Joint Surgery [Am], 66(7):1040-1048, 1984.

12. R. B. Jakob and S. B. Murphy. "Tibial osteotomy for varus gonarthrisis: Indication, planning and operative technique". Instructional Course Lectures, 41:87-93, 1992.

13. J. S. Keene, D. K. Monson, J. M. Roberts, and J. R. Dyreby Jr. "Evaluation of patients for high tibial osteotomy". Clinical Orthopaedics and Related Research, 243:157-165, June 1989.

14. J. L. Knight. "A precision guide-pin technique for wedge and rotatory osteotomy of the femur and tibia". Clinical Orthopaedics and Related Research, 262:248-255, January 1991.

15. T. Koshino, T. Morii, J. Wada, H. Saito, N. Ozawa, and K. Noyori. "High tibial osteotomy with fixation by a blade plate for medial compartment osteoarthritis of the knee". Orthopedic Clinics of North America, 20(2):227-243, April 1989.

16. R. Myrnerts. "Clinical results with the SAAB jig in high tibial osteotomy for medial gonarthrosis". Acta Orthopaedica Scandinavica, 51(3):565-567, June 1980.

17. S. Odenbring, N. Egund, and A. Lindstrand. "A guide instrument for high tibial osteotomy". Acta Orthopaedica Scandinavica, 60(4):449-451, 1989.

18. D. Paley and J. E. Herzenberg. "New concepts in high tibial osteotomy for medial compartment osteoarthritis". Orthopedic Clinics of North America, 25(3):483-498, July 1994.

19. J. F. Rudan and M. A. Simurda. "High tibial osteotomy - A Prospective Clinical and Roentgenographic Review". Clinical Orthopaedics and Related Research, 255:251-256, June 1990.

20. J. F. Rudan and M. A. Simurda. "Valgus high tibial osteotomy - a long-term follow-up study". Clinical Orthopaedics and Related Research, 268:157-160, July 1991.

21. K. Shoemake. "Animating rotation with quaternion curves". Computer Graphics, 19(3):245-254, 1985.

22. K. Shoemake. "Arcball: A user interface for specifying three-dimensional orientation using a mouse". In Proceedings of Graphics Interface, pages 151-156, May 1992.

23. H. Tsumura, S. Himeno, and T. Kawai. "The computer simulation on the correcting osteotomy of osteoarthritis of the knee". Journal of Japanese Orthopaedic Association, 58:565-566, 1984. 